Bangladesh J. Plant Taxon. 28(1): 241-256, 2021 (June)

(C) 2021 Bangladesh Association of Plant Taxonomists

https://doi.org/10.3329/bjpt.v28i1.54220

\title{
FLORISTIC COMPOSITION IN THE RUDERAL AREAS OF SOUTHEAST ANATOLIA, TURKEY
}

\author{
ZEYNEP IZGÖRDÜ AND HASAN AKAN ${ }^{1}$ \\ Depertmant of Biology, Art \& Science Faculty, Harran University, Şanliurfa, Turkey
}

Keywords: Flora; Ruderal plants; Şanlurfa; Anatolia; Turkey.

\begin{abstract}
Survey on the floral diversity is an important activity to evaluate the existing flora. This study was carried out from 2018 to 2020 to investigate the flora existing in the roadside and ruderal areas between Şanlıurfa and Bozova, nearby Euphrates river. A total of 200 taxa belonging to 138 genera and 41 families were determined. 9 of these taxa are endemic to Turkey. Among the plants identified, there are 5 geophytes, 1 parasite and 14 cultivated plants. The families with the most taxa are Fabaceae (47), Asteraceae (30), Lamiaceae (15), Poaceae (13) and Brassicaceae (7). The genera with the dominant taxa are; Astragalus (7), Medicago (7), Trigonella (7), Vicia (5) and Trifolium (5). In distribution of plants in the study area, the Irano-Turanian elements (38\%, 75 taxa) ranks first, Mediterranean elements second (15\%, 30 taxa) and Euro-Siberian elements third $(1 \%, 3)$. The botanical- and vernacular names and habit categories of the recorded plant taxa are cited systematically. Threats to these plants and possible conservation strategies are also discussed briefly.
\end{abstract}

\section{Introduction}

Flora is the list of plant species that have a certain boundary and cover an area. In fact, although the term 'flora' includes the whole plant community, it is generally used for ferns (Pteridophyta) and seed plants (Spermatophyta), i.e. the vascular plants (Güner et al., 2012).

Flora of Turkey, refers to all plant species growing naturally in Turkey. Turkey is one of the very rich and interesting country in the world in terms of its flora due to the facts that it belongs to three different phytogeographical regions, it is rich in geological structure and location and climate diversity, and it houses 9996 plant species of 1320 genera under 167 families, including 3649 endemic taxa (Ekim, 2000; Güner et al., 2012). Peter Hadland Davis, visited Turkey many times, collected a lot of plant samples, and publised the nine volumes of "Flora of Turkey and the East Aegean Islands" in 1965-1985 (Davis, 1965-1985). The 10th volume was published by Davis et al. (1988) and the 11th by Turkish Botanists (Güner et al., 2000). Davis (1965) stated that Turkey serves as a gateway for the spread of Southwest Asian plants to Southern Europe, differentiation area for many species and breeds, it is very rich in terms of endemic species and it is the homeland of many cultivated plants.

Though Turkey is very rich in plant diversity and a gene center of many plant taxa, however, as a result of increasing anthropogenic interactions, many identified and unidentified plant species are rapidly disappearing. Therefore, the importance of floristic studies for collection of updated data on the plant species of this country is increasing day by day for sustainable use and conservation of its plant resources.

South-east Anatolia is a "little known" or "unknown" region of Turkey, with regard to floristic studies (Davis, 1975; Çırpıc1, 1987). The research area falls within this "little known"

\footnotetext{
${ }^{1}$ Corresponding author, email: hakan@harran.edu.tr
} 
region of Turkey. Floristic surveys in Anatolia region were previously done by different researchers, viz. Adıgüzel and Aytaç (2001), Aydoğdu and Akan (2005), Akan et al. (2005), Türkmen et al. (2005), Aslan and Atamov (2006), Parmaksiz et al. (2006), Atamov et al. (2007a), Atamov et al. (2007b), Balos and Akan (2008), Eker et al. (2008), Korkut et al. (2008), Akan and Balos (2008), Doğan (2009), Kaya and Ertekin (2009), Atamov et al. (2009), Cevheri (2011), Abak and Akan (2014), Akan and Ayaz (2016), Şafak (2016), Altay and Karahan (2017), Aslan and Akan (2019), and Yalçınalp and Meral (2019). However, no regular floristic study has been performed in our research area.

"Ruderal plant" is a general name given to plant species that grow in colonies in areas that are not suitable for the life of plants, such as waste areas (Şafak, 2015). Roadside vegetation has various species of different life-forms and origins. Road construction destroys some parts of the natural habitats and as a result, new man-made habitats are formed (Pourrezaei et al., 2017). The structure of the roadside habitat is basically formed by roads and road margins (Frenkel, 1977; Dogan et al., 2004). Roadside habitats differ from surrounding natural areas and support various species with different ecological needs. Roads and roadsides are also included in the ruderal habitat' group (Hamel and Dansereau, 1949), which is an exceptional type of habitat category. Ruderal coenoses develop on walls, ruins, dumps along roadsides and slopes (Poldini, 1992).

Since no study on the floristic composition in the ruderal areas between Şanliurfa and Bozova of southeast Anatolia was known from the literatures, it was thought to be valuable to identity the plant taxa of roadsides ruderals of this area of Turkey, and as the concept of ruderal area is wide, this study has focused on the roadside ruderals only. The objectives of this study were to explore the floristic composition and recognize the endemic and threatened plant species of the roadsides and ruderal areas of the region between Şanlıurfa and Bozova road, nearby Euphrates river, Southeast Anatolia. The data obtained as a result of this study will contribute to the floral diversity of roadsides and ruderal areas of Turkey.

\section{Materials and Methods}

This research was carried out between 2018 and 2020. Our research area belongs to the region between Bozova town and Şanlıurfa province, nearby Euphrates river (Fig. 1). This region is located in C7 square according to Davis's grid system (Davis, 1965) and in the Middle Euphrates section according to Güner et al. (2012). The distance between the two towns is about $50 \mathrm{~km}$. The altitude varies between 500 and $700 \mathrm{~m}$. The settlements around the study area include Kizlar, Günış1k, Korukezen, Tülmen, Küçük Tülmen, Kestaş and Avlak. The area is consisted of 18584 $\mathrm{km}^{2}$ (Güzel, 2020). Şanliurfa province is located in between 37'09'35' N and 38'47'23' E, on the south side of the Southeastern Anatolia Region. Bozova is located in the western part of the province of Şanliurfa, the east and north of the district is mountainous, the south is flat and lower. General vegetation of Bozova district consists of ruderal plants in steppe and flat areas. Şanliurfa has a typical semiarid Mediterranean climate character as it is close to the Euphrates River. Summers are dry and hot, winters are rainy and warm. The average annual temperature of Şanlıurfa is $18.7^{\circ} \mathrm{C}$. Annual rainfall is about $457.8 \mathrm{~mm}$ (Güzel, 2020).

Brown soils constitute a large part of our study area between Şanliurfa and Bozova road. It is the most common soil group. Alluvial soils in the areas carried by the Euphrates River and located along the banks of the Euphrates River (Güzel, 2020). It is suitable for cultivation of various cultivated Plants. In our research area, there are mostly habitats such as roadside, field edge and steppe.

Plant samples (300) were collected during the field studies conducted in the months when the plants coincided with different vegetation periods. These samples were numbered and pressed, 
then dried according to the standard herbarium method and adhered regularly to the cartons (Thiers, 2019). Plant identification were done from Flora of Turkey (Davis, 1965-1985; Davis et al., 1988; Güner et al., 2000). Plant specimens are stored in Harran University Faculty of Arts and Sciences herbarium (HARRAN), Şanlıurfa, Turkey.

Endemic, rare and under more threat to the threat categories of taxa "Turkey Plant Red Data Book" (Ekim, 2000) and is http://www.tehditaltindabitkiler.org.tr/v2/ benefited from the site. The nomenclatural information are given according to The Plant List (2013). Turkish names of plant taxa is given according to List of Vascular Plants of Turkey (Güner et al., 2012).

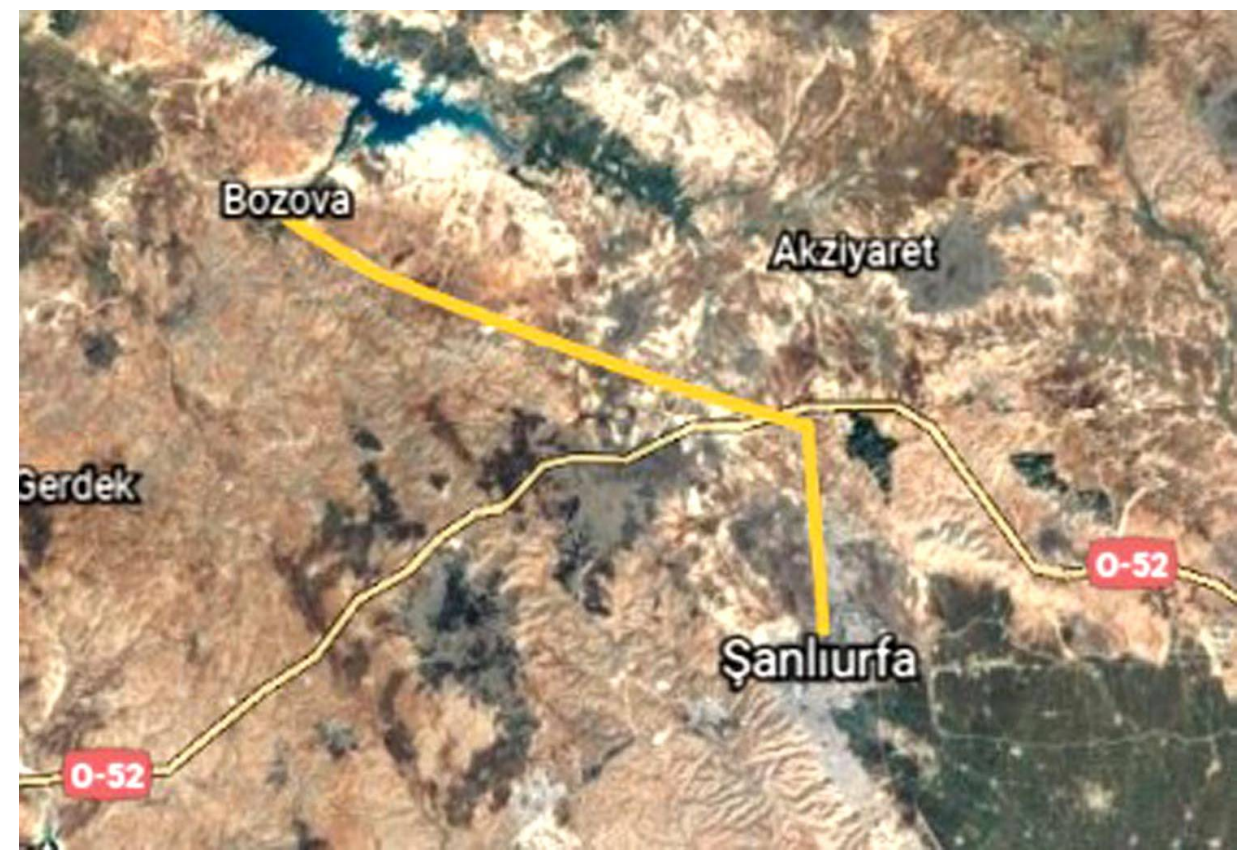

Fig. 1. Geographical map of the research area (Müdürlüğü, 2020).

\section{Results and Discussion}

During this study, a total of 200 taxa belonging to 138 genera and 41 families were found in the roadside ruderals of the area between Şanlıurfa and Bozova of Southeast Anatolia (Table 1). The Gymnospermae of this area is represented by only one species, whereas the Angiospermae by 199 taxa. The dicotyledons of this area consist of $\mathbf{1 7 2}$ under 32 families, and the monocotyledons of under nine families. Most of these taxa belong to the families Fabaceae (47), Asteraceae (30), Lamiaceae (15), Poaceae (13) and Brassicaceae (7). Astragalus, Medicago and Trigonella, with seven species each, and Vicia and Trifolium, with five species each, are are found as the major genera. Each taxon is enlisted with botanical name, vernacular name, family name, habit, citing literature and respective voucher specimen (Table 1).

Total 14 plant species of the area, viz. Pistaci avera, Robinia pseudocacia, Morus alba, M. nigra, Ficus carica, Olea europaea, Pinus nigra, Punica granatum, Persica vulgaris, Amygdalus communis, Amygdalus moriantalis, Rosmarinus officinalis, Pyracantha coccinea and Vitis vinifera, are recognized as cultivated (Table 1). 
Table 1. List of plant taxa recorded from the roadside ruderals of the area between Şanlıurfa and Bozova of Southeast Anatolia.

\begin{tabular}{|c|c|c|c|c|c|c|}
\hline $\begin{array}{l}\text { Sl. } \\
\text { no }\end{array}$ & Family & Botanical name & Habit & Vernacular name & Literature cited & $\begin{array}{l}\text { Voucher } \\
\text { no }\end{array}$ \\
\hline 1 & Amaryllidaceae & $\begin{array}{l}\text { Allium scorodoprasum L. subsp. } \\
\text { rotundum (L.) Steam }\end{array}$ & $\mathrm{H}$ & Delipırasa & $\begin{array}{l}\text { Doğan et al. (2004), } \\
\text { Babacan et al. (2017) }\end{array}$ & Zİ 1117 \\
\hline 2 & Amaranthaceae & Amaranthus albus $\mathrm{L}$. & $\mathrm{H}$ & Kömüşmancarı & $\begin{array}{l}\text { Davis (1965-1988), } \\
\text { Polunin (1997), } \\
\text { Doğan et al. (2004) }\end{array}$ & Zİ 1145 \\
\hline 3 & Anacardiaceae & *Pistacia vera $\mathrm{L}$. & $\mathrm{T}$ & Antepfistığı & - & Zİ 1207 \\
\hline 4 & Anacardiaceae & Rhus coriaria $\mathrm{L}$. & $S$ & Sumak & Doğan et al, (2004) & Zİ 1205 \\
\hline 5 & Apiaceae & Artedia squamata $\mathrm{L}$. & $\mathrm{H}$ & Karabenek & Babacan et al. (2017) & $\begin{array}{l}\text { ZI } 1066 \\
\text { ZI } 1101 \\
\text { ZI } 1182\end{array}$ \\
\hline 6 & Apiaceae & Coriandrum sativum $\mathrm{L}$. & $\mathrm{H}$ & Kişniş & Doğan et al, (2004) & ZI 1170 \\
\hline 7 & Apiaceae & Pimpinella corymbosa Boiss. & $\mathrm{H}$ & Salkımanason & Yeşil et al. (2018) & ZI 1234 \\
\hline 8 & Apiaceae & $\begin{array}{l}\text { Pimpinella eriocarpos Banks \& } \\
\text { Sol }\end{array}$ & $\mathrm{H}$ & Meyane & Yeşil et al. (2018) & ZI 1095 \\
\hline 9 & Apiaceae & Scandix iberica M. Bieb & $\mathrm{H}$ & Atkişnekotu & $\begin{array}{l}\text { Kerar \& Akan } \\
\text { (2019) }\end{array}$ & ZI 1244 \\
\hline 10 & Apiaceae & Tordylium hasselquistiae DC. & $\mathrm{H}$ & Ekindavulotu & - & ZI 1103 \\
\hline 11 & Apiaceae & Torilis leptophylla (L.) Rchb. F. & $\mathrm{H}$ & İncedercikotu & $\begin{array}{l}\text { Ertekin (2002), } \\
\text { Babacan } \text { et al. (2017) }\end{array}$ & ZI 1051 \\
\hline 12 & Araceae & $\begin{array}{l}\text { Biarum carduchorum (Schott) } \\
\text { Engl. }\end{array}$ & $\mathrm{H}$ & Kardi & - & ZI 1142 \\
\hline 13 & Asparagaceae & $\begin{array}{l}\text { Hyacinthella nervosa (Bertol.) } \\
\text { Chouard }\end{array}$ & $\mathrm{H}$ & Arapkopçası & - & ZI 1000 \\
\hline 14 & Asparagaceae & Muscari comosum (L.) Mill. & $\mathrm{H}$ & Morbaş & - & ZI 1012 \\
\hline 15 & Asparagaceae & Muscari neglectum Guss. ex Ten & $\mathrm{H}$ & Arapüzümü & - & ZI1001 \\
\hline 16 & Asparagaceae & Ornithogalum narbonense $\mathrm{L}$. & $\mathrm{H}$ & Akbaldır & Davis (1965-1988) & ZI 1118 \\
\hline 17 & Asteraceae & $\begin{array}{l}\text { Achillea aleppica DC. } \\
\text { subsp. aleppica }\end{array}$ & $\mathrm{H}$ & Tatarciotu & Abak \& Akan (2014) & ZI 1004 \\
\hline 18 & Asteraceae & Anthemis hyalina DC. & $\mathrm{H}$ & Dermanpapatyası & Abak \& Akan (2014) & ZI 1107 \\
\hline 19 & Asteraceae & Anthemis pungens Yavin & $\mathrm{H}$ & Geyikpapatyası & Abak \& Akan (2014) & ZI 1038 \\
\hline 20 & Asteraceae & Calendula arvensis (Vaill.) L. & $\mathrm{H}$ & Portakalnergisi & $\begin{array}{l}\text { Davis (1965-1988), } \\
\text { Doğan et al. (2004) }\end{array}$ & ZI 1025 \\
\hline 21 & Asteraceae & Carduus nutans L. subsp. nutans & $\mathrm{H}$ & Eşekdikeni & Abak \& Akan (2014) & ZI 1108 \\
\hline 22 & Asteraceae & $\begin{array}{l}\text { Carduus pycnocephalus L. subsp. } \\
\text { breviphyllarius } \text { P.H. Davis }\end{array}$ & $\mathrm{H}$ & Kilindor & Abak \& Akan (2014) & ZI 1048 \\
\hline 23 & Asteraceae & Carthamus lanatus $\mathrm{L}$. & $\mathrm{H}$ & Sarıdiken & Abak \& Akan (2014) & ZI 1153 \\
\hline 24 & Asteraceae & $\begin{array}{l}\text { Centaurea iberica Trev. ex } \\
\text { Sprengel }\end{array}$ & $\mathrm{H}$ & Deligözdikeni & $\begin{array}{l}\text { Davis (1965-1988), } \\
\text { Akan et al. (2005), } \\
\text { Abak \& Akan (2014) }\end{array}$ & ZI 1138 \\
\hline 25 & Asteraceae & Centaurea rigida Banks \& Sol. & $\mathrm{H}$ & Gürbüzdikeni & Abak \& Akan (2014) & ZI 1219 \\
\hline
\end{tabular}


Table 1 contd.

\begin{tabular}{|c|c|c|c|c|c|c|}
\hline $\begin{array}{l}\text { Sl. } \\
\text { no. }\end{array}$ & Family & Botanical name & Habit & Vernacular name & Literature cited & $\begin{array}{l}\text { Voucher } \\
\text { no. }\end{array}$ \\
\hline 26 & Asteraceae & $\begin{array}{l}\text { Centaurea solstitialis L. subsp. } \\
\text { solstitialis }\end{array}$ & $\mathrm{H}$ & Çakırdikeni & $\begin{array}{l}\text { Davis (1965-1988), } \\
\text { Poldini (1992), } \\
\text { Doğan et al. (2004), } \\
\text { Abak \& Akan } \\
\text { (2014), Babacan et } \\
\text { al. (2017) }\end{array}$ & ZI 1204 \\
\hline 27 & Asteraceae & Chardinia orientalis (L.) Kuntze. & $\mathrm{H}$ & Çağlaotu & $\begin{array}{l}\text { Davis (1965-1988), } \\
\text { Abak \& Akan (2014) }\end{array}$ & ZI 1052 \\
\hline 28 & Asteraceae & Cichorium intybus L. & $\mathrm{H}$ & Hindiba & $\begin{array}{l}\text { Davis (1965-1988), } \\
\text { Ozturk et al. } \\
\text { (1990), Ozturk \& } \\
\text { Ozcelik (1991), } \\
\text { Ertekin (2002), } \\
\text { Doğan et al, (2004), } \\
\text { Abak \& Akan } \\
\text { (2014) }\end{array}$ & ZI 1221 \\
\hline 29 & Asteraceae & Conyza canadensis (L.) Cronquist & $\mathrm{H}$ & Selviotu & $\begin{array}{l}\text { Abak \& Akan } \\
\text { (2014), Ertekin } \\
\text { (2002) }\end{array}$ & ZI 1143 \\
\hline 30 & Asteraceae & Cota altissima (L.) J. Gay & $\mathrm{H}$ & Köpekpapatyası & $\begin{array}{l}\text { Abak \& Akan } \\
\text { (2014), Babacan et } \\
\text { al. (2017) }\end{array}$ & ZI 1083 \\
\hline 31 & Asteraceae & Crepis sancta (L.) Bornm. & $\mathrm{H}$ & Yabankıskısı & Abak \& Akan (2014) & $\begin{array}{l}\text { ZI } 1041 \\
\text { ZI } 1140\end{array}$ \\
\hline 32 & Asteraceae & Crupina crupinastrum (Moris) Vis. & $\mathrm{H}$ & Gelindöndüren & Babacan et al. (2017) & $\begin{array}{l}\text { ZI } 1080 \\
\text { ZI } 1049\end{array}$ \\
\hline 33 & Asteraceae & $\begin{array}{l}\text { Cyanus depressus (M. Bieb.) } \\
\text { Sojak. }\end{array}$ & $\mathrm{H}$ & Gökbaş & Babacan et al. (2017) & $\begin{array}{l}\text { ZI } 1096 \\
\text { ZI } 1192\end{array}$ \\
\hline 34 & Asteraceae & $\begin{array}{l}\text { Echinops spinosissimus Turra } \\
\text { subsp. spinosissimus }\end{array}$ & $\mathrm{H}$ & Eşekköftesi & Davis (1965-1988) & ZI 1132 \\
\hline 35 & Asteraceae & Filago pyramidata L. & $\mathrm{H}$ & Ateşpamuğu & $\begin{array}{l}\text { Davis (1965-1988), } \\
\text { Doğan et al. (2004), } \\
\text { Abak \& Akan (2014) }\end{array}$ & ZI 1199 \\
\hline 36 & Asteraceae & $\begin{array}{l}\text { Geropogon hybridus (L.) } \\
\text { SchultzBip. }\end{array}$ & $\mathrm{H}$ & Melezyemlik & - & $\begin{array}{l}\text { ZI } 1026 \\
\text { ZI } 1036\end{array}$ \\
\hline 37 & Asteraceae & $\begin{array}{l}\text { Gundelia armata } \\
\text { (Freyn \& Sint.) Firat }\end{array}$ & $\mathrm{H}$ & Haskenger & Abak \& Akan (2014) & ZI 1109 \\
\hline 38 & Asteraceae & Notobasis syriaca (L.) Cass. & $\mathrm{H}$ & $\begin{array}{l}\text { Yavan } \\
\text { kenger }\end{array}$ & Davis (1965-1988), & $\begin{array}{l}\text { ZI } 1187 \\
\text { ZI } 1185\end{array}$ \\
\hline 39 & Asteraceae & $\begin{array}{l}\text { Onopordum carduchorum } \\
\text { Bornm\&Beauverd }\end{array}$ & $\mathrm{H}$ & Kavdikeni & Balos \& Akan (2008) & ZI 1136 \\
\hline 40 & Asteraceae & Senecio vernalis Waldst. \& Kit. & $\mathrm{H}$ & $\begin{array}{l}\text { Kanarya } \\
\text { otu }\end{array}$ & $\begin{array}{l}\text { Davis (1965-1988), } \\
\text { Ozturk et al.(1990), } \\
\text { Ertekin (2002), } \\
\text { Doğan et al. (2004), } \\
\text { Aydoğdu \& Akan } \\
\text { (2005), Abak \& } \\
\text { Akan (2014) }\end{array}$ & $\begin{array}{l}\text { ZI } 1039 \\
\text { ZI } 1164 \\
\text { ZI } 1165 \\
\text { ZI } 1208\end{array}$ \\
\hline 41 & Asteraceae & Siebera nana (DC.) Bornm & $\mathrm{H}$ & Bodurfezaçiçeği & $\begin{array}{l}\text { Tugay \& Öztürk } \\
(2003)\end{array}$ & ZI 1127 \\
\hline 42 & Asteraceae & Scorzonera kotschyi Boiss. & $\mathrm{H}$ & $\begin{array}{l}\text { Nurteke } \\
\text { sakali }\end{array}$ & - & ZI 1056 \\
\hline
\end{tabular}


Table 1 contd.

\begin{tabular}{|c|c|c|c|c|c|c|}
\hline $\begin{array}{l}\text { Sl. } \\
\text { no. }\end{array}$ & Family & Botanical name & Habit & Vernacular name & Literature cited & $\begin{array}{l}\text { Voucher } \\
\text { no. }\end{array}$ \\
\hline 43 & Asteraceae & $\begin{array}{l}\text { Scorzonera laciniata L. subsp. } \\
\text { laciniata }\end{array}$ & $\mathrm{H}$ & Parım & - & $\begin{array}{l}\text { ZI } 1050 \\
\text { ZI } 1186\end{array}$ \\
\hline 44 & Asteraceae & $\begin{array}{l}\text { Tragopogon porrifolius L. subsp. } \\
\text { longirostris (Sch. Bip.) Greuter }\end{array}$ & $\mathrm{H}$ & Helevan & $\begin{array}{l}\text { Davis (1965-1988), } \\
\text { Doğan et al. }(2004)\end{array}$ & $\begin{array}{l}\text { ZI } 1097 \\
\text { ZI } 1232\end{array}$ \\
\hline 45 & Asteraceae & $\begin{array}{l}\text { Xanthium strumarium L. subsp. } \\
\text { strumarium }\end{array}$ & $\mathrm{H}$ & Kocapitrak & $\begin{array}{l}\text { Ozturk et al. (1990), } \\
\text { Ertekin (2002), } \\
\text { Doğan et al. (2004), } \\
\text { Abak \& Akan (2014) }\end{array}$ & ZI 1150 \\
\hline 46 & Asteraceae & Zoegea leptaurea $\mathrm{L}$. & $\mathrm{H}$ & Sarıdüğme & Doğan (2009) & ZI 1200 \\
\hline 47 & Brassicaceae & Alyssum strictum Willd. & $\mathrm{H}$ & Dikkuduzotu & Doğan (2009) & ZI 1023 \\
\hline 48 & Brassicaceae & $\begin{array}{l}\text { Capsella bursa-pastoris (L.) } \\
\text { Medik. }\end{array}$ & $\mathrm{H}$ & $\begin{array}{l}\text { Çoban } \\
\text { çantası }\end{array}$ & Doğan (2009) & ZI 1160 \\
\hline 49 & Brassicaceae & Clypeola jonthlaspi $\mathrm{L}$. & $\mathrm{H}$ & Akçeotu & Acar (2001) & ZI1022 \\
\hline 50 & Brassicaceae & Eruca vesicaria (L.) Cav. & $\mathrm{H}$ & Roka & - & ZI 1176 \\
\hline 51 & Brassicaceae & Lepidium draba $\mathrm{L}$. & $\mathrm{H}$ & Diğnik & $\begin{array}{l}\text { Balos \& Akan } \\
(2008), \text { Babacan } \\
\text { et al. }(2017)\end{array}$ & ZI 1236 \\
\hline 52 & Brassicaceae & Sinapis alba L. subsp alba & $\mathrm{H}$ & Mamalık & $\begin{array}{l}\text { Davis (1965-1988), } \\
\text { Doğan et al. (2004) }\end{array}$ & ZI 1027 \\
\hline 53 & Brassicaceae & Sinapis arvensis $\mathrm{L}$. & $\mathrm{H}$ & Hardal & $\begin{array}{l}\text { Davis (1965-1988), } \\
\text { Ozturk \& Ozcelik } \\
\text { (1991), Ertekin } \\
\text { (2002), Doğan } \text { et al, } \\
\text { (2004) }\end{array}$ & ZI 1172 \\
\hline 54 & Boraginaceae & Alkanna strigosa Boiss. \&Hohen. & $\mathrm{H}$ & Havacivaotu & $\begin{array}{l}\text { Yıldırım et al. } \\
\text { (2016), Aydoğdu \& } \\
\text { Akan (2005), } \\
\text { Babacan } \text { et al. (2017) }\end{array}$ & $\begin{array}{l}\text { ZI } \\
1044\end{array}$ \\
\hline 55 & Boraginaceae & Anchusa azurea Mill. var. azurea & $\mathrm{H}$ & Sığırdili & Babacan et al. (2017) & $\begin{array}{l}\text { ZI } 1092 \\
\text { ZI } 1058\end{array}$ \\
\hline 56 & Boraginaceae & $\begin{array}{l}\text { Anchusa azurea Mill. var. kurdica } \\
\text { (Guşul.) Chamb. }\end{array}$ & $\mathrm{H}$ & & $\begin{array}{l}\text { Bozok \& Aksoy } \\
\text { (2013) }\end{array}$ & $\begin{array}{l}\text { ZI } 1146 \\
\text { ZI } 1139\end{array}$ \\
\hline 57 & Boraginaceae & Heliotropium dolosum De Not. & $\mathrm{H}$ & Balbulotu & Davis (1965-1988) & ZI 1228 \\
\hline 58 & Boraginaceae & $\begin{array}{l}\text { Rochelia disperma (L.f.) K. Koch } \\
\text { var. disperma }\end{array}$ & $\mathrm{H}$ & Kuşçırnağ 1 & 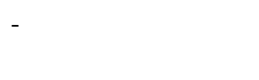 & ZI 1074 \\
\hline 59 & Capparaceae & $\begin{array}{l}\text { Capparis sicula Veill. subsp. } \\
\text { sicula }\end{array}$ & S & Delikarpuzu & $\begin{array}{l}\text { Akan et al. (2004), } \\
\text { Balos \& Akan (2008) }\end{array}$ & $\begin{array}{l}\text { ZI } 1148 \\
\text { ZI } 1223\end{array}$ \\
\hline 60 & Caprifoliaceae & Cephalaria syriaca (L.) Schrad & $\mathrm{H}$ & Pelemir & $\begin{array}{l}\text { Ertekin (2002), } \\
\text { Babacan et al. (2017) }\end{array}$ & ZI 1099 \\
\hline 61 & Caprifoliaceae & Scabiosa calocephala Boiss. & $\mathrm{H}$ & Çayıruyuzotu & $\begin{array}{l}\text { Davis (1965-1988), } \\
\text { Doğan et al, (2004) }\end{array}$ & ZI 1130 \\
\hline 62 & Caprifoliaceae & Scabiosa persica Boiss. & $\mathrm{H}$ & Acemzivanı & - & ZI 1093 \\
\hline 63 & Caprifoliaceae & $\begin{array}{l}\text { Valerianella vesicaria }(\mathrm{L} .) \\
\text { Moench }\end{array}$ & $\mathrm{H}$ & Kuzugevreği & - & $\begin{array}{l}\text { ZI } 1045 \\
\text { ZI } 1065\end{array}$ \\
\hline 64 & Caprifoliaceae & Valerianella pumila (L.) DC. & $\mathrm{H}$ & Bağkuzugevreği & - & ZI 1105 \\
\hline
\end{tabular}


Table 1 contd.

\begin{tabular}{|c|c|c|c|c|c|c|}
\hline $\begin{array}{l}\text { Sl. } \\
\text { no. }\end{array}$ & Family & Botanical name & Habit & Vernacular name & Literature cited & $\begin{array}{l}\text { Voucher } \\
\text { no. }\end{array}$ \\
\hline 65 & Caryophyllaceae & $\begin{array}{l}\text { Arenaria sabulinea Griseb. ex } \\
\text { Fenzl }\end{array}$ & $\mathrm{H}$ & Firatkumotu & - & ZI 1163 \\
\hline 66 & Caryophyllaceae & Dianthus strictus Bank \& Sol. & $\mathrm{H}$ & Dimisok & - & ZI 1227 \\
\hline 67 & Caryophyllaceae & Minuartia montana $\mathrm{L}$. & $\mathrm{H}$ & Demettıstısı & - & ZI 1239 \\
\hline 68 & Caryophyllaceae & Silene coniflora Nees ex Otth & $\mathrm{H}$ & Çölnakılı & Davis (1965-1988) & $\begin{array}{l}\text { ZI } 1030 \\
\text { ZI } 1070\end{array}$ \\
\hline 69 & Caryophyllaceae & Silene crassipes Fenzl & $\mathrm{H}$ & Tarlanakılı & - & ZI 1235 \\
\hline 70 & Caryophyllaceae & $\begin{array}{l}\text { Vaccaria hispanica (Mill.) } \\
\text { Rauschert }\end{array}$ & $\mathrm{H}$ & Ekinebesi & - & ZI 1224 \\
\hline 71 & Convolvulaceae & Convolvulus arvensis $\mathrm{L}$. & $\mathrm{Cl}$ & Tarlasarmaşı $\breve{g} 1$ & $\begin{array}{l}\text { Davis (1965-1988), } \\
\text { Polunin (1997), } \\
\text { Doğan et al.(2004) }\end{array}$ & $\begin{array}{l}\text { ZI } 1128 \\
\text { ZI } 1217 \\
\text { ZI } 1231\end{array}$ \\
\hline 72 & Convolvulaceae & $\begin{array}{l}\text { Convolvulus dorycnium L. subsp. } \\
\text { dorycnium }\end{array}$ & $\mathrm{Cl}$ & Bağarcıkurganı & - & ZI 1125 \\
\hline 73 & Convolvulaceae & $\begin{array}{l}\text { Convolvulus galaticus Rost. ex } \\
\text { Choisy }\end{array}$ & $\mathrm{Cl}$ & Bozsarmaşık & Ertekin (2002) & $\begin{array}{l}\text { ZI } 1054 \\
\text { ZI } 1230\end{array}$ \\
\hline 74 & Crassulaceae & Umbilicus horizontalis DC. & $\mathrm{H}$ & Kalaba & - & ZI 1154 \\
\hline 75 & Cyperaceae & Cyperus glaber $\mathrm{L}$. & $\mathrm{H}$ & Küsnüotu & - & ZI 1177 \\
\hline 76 & Cyperaceae & Cyperus longus L. subsp. longus & $\mathrm{H}$ & Karatopalak & - & ZI 1218 \\
\hline 77 & Euphorbiaceae & Euphorbia aleppica $\mathrm{L}$. & $\mathrm{H}$ & Haşul & Davis (1965-1988) & ZI 1071 \\
\hline 78 & Euphorbiaceae & $\begin{array}{l}\text { Euphorbia cheiradenia Boiss. \& } \\
\text { Hohen }\end{array}$ & $\mathrm{H}$ & Şirker & Babacan et al. (2017) & $\begin{array}{l}\text { ZI } 1215 \\
\text { ZI } 1157 \\
\text { ZI } 1135 \\
\text { ZI } 1133\end{array}$ \\
\hline 79 & Euphorbiaceae & $\begin{array}{l}\text { Euphorbia falcata L. subsp. } \\
\text { falcata }\end{array}$ & $\mathrm{H}$ & Eğrisütleğen & Babacan et al. (2017) & $\begin{array}{l}\text { ZI } 1094 \\
\text { ZI } 1089\end{array}$ \\
\hline 80 & Euphorbiaceae & $\begin{array}{l}\text { Euphorbia helioscopia L. subsp. } \\
\text { helioscopia }\end{array}$ & $\mathrm{H}$ & Feribanotu & - & ZI 1174 \\
\hline 81 & Fabaceae & Astragalus aduncus Willd. & $\mathrm{H}$ & Çengelgeven & Ekici et al. (2015) & ZI 1110 \\
\hline 82 & Fabaceae & Astragalus aleppicus Boiss. & $\mathrm{H}$ & Halepgeveni & - & ZI 1246 \\
\hline 83 & Fabaceae & $\begin{array}{l}\text { Astragalus caprinus L. subsp. } \\
\text { caprinus }\end{array}$ & $\mathrm{H}$ & Tekegeveni & - & ZI 1002 \\
\hline 84 & Fabaceae & Astragalus scabrifolius Boiss. & $\mathrm{H}$ & Gövdesizgeven & - & ZI 1005 \\
\hline 85 & Fabaceae & $\begin{array}{l}\text { Astragalus suberosus Banks \& } \\
\text { Sol. }\end{array}$ & $\mathrm{H}$ & Yemenigeveni & Babacan et al. (2017) & ZI 1003 \\
\hline 86 & Fabaceae & Astragalus triradiatus Bunge. & $\mathrm{H}$ & Üçgeven & - & ZI 1028 \\
\hline 87 & Fabaceae & $\begin{array}{l}\text { Astragalus xylobasis } \\
\text { Freyn\&Bornm. }\end{array}$ & $\mathrm{H}$ & Kemaliyegeveni & Ekici et al. (2015) & ZI 1111 \\
\hline 88 & Fabaceae & $\begin{array}{l}\text { Coronilla scorpioides (L.) W.D.J. } \\
\text { Koch }\end{array}$ & $\mathrm{H}$ & Akrepburçağı & Babacan et al. (2017) & ZI 1031 \\
\hline 89 & Fabaceae & Glycyrrhiza glabra L. var. glabra & $\mathrm{H}$ & Meyan & $\begin{array}{l}\text { Ertekin (2002), } \\
\text { Aydoğdu \& Akan } \\
(2005), \text { Babacan } e t \\
\text { al. (2017), Balos \& } \\
\text { Akan (2008) }\end{array}$ & $\begin{array}{l}\text { ZI } 1061 \\
\text { ZI } 1178\end{array}$ \\
\hline 90 & Fabaceae & Hedysarum varium Willd. & $\mathrm{H}$ & Batalak & - & ZI 1081 \\
\hline
\end{tabular}


Table 1 contd.

\begin{tabular}{|c|c|c|c|c|c|c|}
\hline $\begin{array}{l}\text { Sl. } \\
\text { no. }\end{array}$ & Family & Botanical name & Habit & Vernacular name & Literature cited & $\begin{array}{l}\text { Voucher } \\
\text { no. }\end{array}$ \\
\hline 91 & Fabaceae & $\begin{array}{l}\text { Hymenocarpos circinnatus (L.) } \\
\text { Savi }\end{array}$ & $\mathrm{H}$ & Pulluot & - & ZI 1085 \\
\hline 92 & Fabaceae & Lathyrus pseudo-cicera Pamp. & $\mathrm{H}$ & Hatunbaklası & - & ZI 1046 \\
\hline 93 & Fabaceae & Lens culinaris Medik. & $\mathrm{H}$ & Mercimek & - & ZI 1225 \\
\hline 94 & Fabaceae & Lotus gebelia Vent. var. gebelia & $\mathrm{H}$ & Gülgazalboynuzu & - & ZI 1114 \\
\hline 95 & Fabaceae & $\begin{array}{l}\text { Medicago crassipes (Boiss.) E. } \\
\text { Small }\end{array}$ & $\mathrm{H}$ & Hançeryoncası & Akan et al. (2009) & ZI 1011 \\
\hline 96 & Fabaceae & $\begin{array}{l}\text { Medicago fischeriana (Ser.) } \\
\text { Trautv. }\end{array}$ & $\mathrm{H}$ & Mizrakyonca & Akan et al. (2009) & ZI 1008 \\
\hline 97 & Fabaceae & $\begin{array}{l}\text { Medicago monantha (C.A. Mey.) } \\
\text { Trautv }\end{array}$ & $\mathrm{H}$ & Dağgurniği & Akan et al. (2009) & $\begin{array}{l}\text { ZI } 1007 \\
\text { ZI } 1195\end{array}$ \\
\hline 98 & Fabaceae & $\begin{array}{l}\text { Medicago phrygia (Boiss. \& } \\
\text { Balansa) E. Small }\end{array}$ & $\mathrm{H}$ & Uşakyoncası & Akan et al. (2009) & ZI 1010 \\
\hline 99 & Fabaceae & $\begin{array}{l}\text { Medicago polymorpha L. var. } \\
\text { polymorpha }\end{array}$ & $\mathrm{H}$ & Kırkyonca & $\begin{array}{l}\text { Polunin (1997), } \\
\text { Doğan et al. (2004), } \\
\text { Balos \& Akan (2008) }\end{array}$ & ZI 1033 \\
\hline 100 & Fabaceae & Medicago radiata $\mathrm{L}$. & $\mathrm{H}$ & Hilalyonca & - & ZI 1040 \\
\hline 101 & Fabaceae & Medicago x varia Martyn & $\mathrm{H}$ & Yabanyoncası & - & ZI 1006 \\
\hline 102 & Fabaceae & Melilotus indicus (L.) All. & $\mathrm{H}$ & Otuzluyonca & - & ZI 1112 \\
\hline 103 & Fabaceae & Melilotus officinalis (L.) Desr. & $\mathrm{H}$ & Kokuluyonca & $\begin{array}{l}\text { Balos \&Akan (2008), } \\
\text { Babacan et al. (2017) }\end{array}$ & ZI 1180 \\
\hline 104 & Fabaceae & $\begin{array}{l}\text { Onobrychis aequidentata (Sibth. } \\
\& \text { Sm.) d Urv }\end{array}$ & $\mathrm{H}$ & Dişlekkorunga & - & ZI 1084 \\
\hline 105 & Fabaceae & Onobrychis caput-galli (L.) Lam. & $\mathrm{H}$ & Pitrakkorunga & $\begin{array}{l}\text { Davis (1965-1988), } \\
\text { Polunin (1997), } \\
\text { Doğan et al. (2004) }\end{array}$ & ZI 1113 \\
\hline 106 & Fabaceae & Onobrychis galegifolia Boiss. & $\mathrm{H}$ & Darpkorungası & - & ZI 1216 \\
\hline 107 & Fabaceae & $\begin{array}{l}\text { Pisum sativum } \mathrm{L} \text {. subsp. sativum } \\
\text { var. arvense }(\mathrm{L} \text {.) Poiret }\end{array}$ & $\mathrm{H}$ & Bezelye & $\begin{array}{l}\text { Ertekin (2002), } \\
\text { Babacan et al. (2017) }\end{array}$ & ZI 1062 \\
\hline 108 & Fabaceae & $\begin{array}{l}\text { Prosopis farcta (Banks\& Sol.) J.F. } \\
\text { Macbr. }\end{array}$ & $\mathrm{H}$ & Çediotu & - & $\begin{array}{l}\text { ZI } 1152 \\
\text { ZI } 1201\end{array}$ \\
\hline 109 & Fabaceae & *Robinia pseudocacia $\mathrm{L}$. & $\mathrm{T}$ & Yalanciakasya & Davis (1965-1988) & ZI 1212 \\
\hline 110 & Fabaceae & $\begin{array}{l}\text { Scorpiurus subvillosus L. var. } \\
\text { subvillosus }\end{array}$ & $\mathrm{H}$ & Koyundücüğü & - & ZI 1086 \\
\hline 111 & Fabaceae & $\begin{array}{l}\text { Trifolium nigrescens Viv. subsp. } \\
\text { nigrescens }\end{array}$ & $\mathrm{H}$ & Yanıküçgül & Babacan et al. (2017) & ZI 1073 \\
\hline 112 & Fabaceae & Trifolium purpureum Lois. & $\mathrm{H}$ & Morüçgül & Davis (1965-1988) & $\begin{array}{l}\text { ZI } 1102 \\
\text { ZI } 1104\end{array}$ \\
\hline 113 & Fabaceae & $\begin{array}{l}\text { Trifolium resupinatum } \mathrm{L} \text {. var. } \\
\text { resupinatum }\end{array}$ & $\mathrm{H}$ & Anadoluüçgülü & $\begin{array}{l}\text { Davis (1965-1988) } \\
\text { Doğan et al, (2004) }\end{array}$ & ZI 1079 \\
\hline 114 & Fabaceae & Trifolium spumosum L. & $\mathrm{H}$ & Keseyonca & Davis (1965-1988) & ZI 1064 \\
\hline 115 & Fabaceae & $\begin{array}{l}\text { Trifolium stellatum } \mathrm{L} . \text { var. } \\
\text { stellatum }\end{array}$ & $\mathrm{H}$ & Yildızyonca & $\begin{array}{l}\text { Davis (1965-1988), } \\
\text { Doğan et al, (2004) }\end{array}$ & $\begin{array}{l}\text { ZI } 1019 \\
\text { ZI } 1082 \\
\text { ZI } 1075\end{array}$ \\
\hline
\end{tabular}


Table 1 contd.

\begin{tabular}{|c|c|c|c|c|c|c|}
\hline $\begin{array}{l}\text { Sl. } \\
\text { no. }\end{array}$ & Family & Botanical name & Habit & Vernacular name & Literature cited & $\begin{array}{l}\text { Voucher } \\
\text { no. }\end{array}$ \\
\hline 116 & Fabaceae & Trigonella caelesyriaca Boiss. & $\mathrm{H}$ & Handekok & Akan et al. (2009) & ZI 1016 \\
\hline 117 & Fabaceae & Trigonella filipes Boiss. & $\mathrm{H}$ & İnceboyotu & Akan et al. (2009) & ZI 1035 \\
\hline 118 & Fabaceae & Trigonella kotschyi Fenzl. & $\mathrm{H}$ & Akboyotu & Akan et al. (2009) & ZI 1009 \\
\hline 119 & Fabaceae & $\begin{array}{l}\text { Trigonella mesopotamica Hub.- } \\
\text { Mor. }\end{array}$ & $\mathrm{H}$ & Dicleboyotu & Akan et al. (2009) & ZI 1245 \\
\hline 120 & Fabaceae & Trigonella monspeliaca $\mathrm{L}$. & $\mathrm{H}$ & Somçemenotu & Akan et al. (2009) & ZI 1196 \\
\hline 121 & Fabaceae & Trigonella spicata Sibth. \&Sm. & $\mathrm{H}$ & Başakboyutu & Akan et al. (2009) & ZI 1226 \\
\hline 122 & Fabaceae & Trigonella spruneriana Boiss. & $\mathrm{H}$ & Koçboyotu & Akan et al. (2009) & $\begin{array}{l}\text { ZI } 1223 \\
\text { ZI } 1018\end{array}$ \\
\hline 123 & Fabaceae & Vicia hybrida $\mathrm{L}$. & $\mathrm{H}$ & Melezbakla & - & ZI 1060 \\
\hline 124 & Fabaceae & $\begin{array}{l}\text { Vicia narbonensis L. var. } \\
\text { narbonensis }\end{array}$ & $\mathrm{H}$ & Kocaçı̆̆ & - & $\begin{array}{l}\text { ZI } 1032 \\
\text { ZI } 1072\end{array}$ \\
\hline 125 & Fabaceae & Vicia palaestina Boiss. & $\mathrm{H}$ & Yabaniküşve & - & ZI 1015 \\
\hline 126 & Fabaceae & Vicia peregrina $\mathrm{L}$. & $\mathrm{H}$ & Kavli & Balos \& Akan (2008) & ZI 1017 \\
\hline 127 & Fabaceae & Vicia sativa L. subsp. sativa & $\mathrm{H}$ & Fiğ & $\begin{array}{l}\text { Frenkel (1977), } \\
\text { Doğan et al. (2004), } \\
\text { Balos \& Akan (2008) }\end{array}$ & ZI 1043 \\
\hline 128 & Geraniaceae & Geranium tuberosum L. & $\mathrm{H}$ & Çakmuz & $\begin{array}{l}\text { Eker } \text { et al. (2008), } \\
\text { Babacan et al. (2017) }\end{array}$ & $\begin{array}{l}\text { ZI } 1068 \\
\text { ZI } 1175\end{array}$ \\
\hline 129 & Geraniaceae & $\begin{array}{l}\text { Erodium cicutarium (L.) L Hér. } \\
\text { subsp. cicutarium }\end{array}$ & $\mathrm{H}$ & İğnelik & $\begin{array}{l}\text { Frenkel (1977), } \\
\text { Polunin (1997), } \\
\text { Doğan et al. (2004), } \\
\text { Aydoğdu \& Akan } \\
(2005)\end{array}$ & $\begin{array}{l}\text { ZI } 1020 \\
\text { ZI } 1169\end{array}$ \\
\hline 130 & Hypericaceae & Hypericum perforatum $\mathrm{L}$. & $\mathrm{H}$ & Kantaron & - & ZI 1115 \\
\hline 131 & Hyperıcaceae & Hypericum triquetrifolium Turra & $\mathrm{H}$ & Pirpirotu & - & ZI 1155 \\
\hline 132 & Iridaceae & $\begin{array}{l}\text { Crocus cancellatus Herb. subsp. } \\
\text { damascenus (Herb.) B. Mathew }\end{array}$ & $\mathrm{H}$ & Pivok & - & ZI 1144 \\
\hline 133 & Iridaceae & Iris persica $\mathrm{L}$. & $\mathrm{H}$ & Buzala & - & ZI 1171 \\
\hline 134 & Iridaceae & Iris $x$ germanica $\mathrm{L}$. & $\mathrm{H}$ & Göksüsen & - & ZI 1100 \\
\hline 135 & Ixioliriaceae & $\begin{array}{l}\text { Ixiolirion tataricum (Pall.) Schult. } \\
\& \text { Schult. F. var. tataricum }\end{array}$ & $\mathrm{H}$ & Köpekotu & - & ZI 1241 \\
\hline 136 & Juncaceae & Juncus inflexus L. & $\mathrm{H}$ & Sazak & $\begin{array}{l}\text { Davis (1965-1988), } \\
\text { Doğan et al. }(2004), \\
\text { Babacan et al. }(2017)\end{array}$ & ZI 1243 \\
\hline 137 & Lamiaceae & $\begin{array}{l}\text { Ballota saxatilis } \text { Siebernex C. } \\
\text { Presl subsp. saxatilis }\end{array}$ & $\mathrm{H}$ & Nemnemotu & $\begin{array}{l}\text { Aydoğdu \& Akan } \\
(2005)\end{array}$ & ZI 1149 \\
\hline 138 & Lamiaceae & $\begin{array}{l}\text { Lamium amplexicaule } \\
\text { L.var.aleppicum (Boiss. et } \\
\text { Hausskn) Bornm. }\end{array}$ & $\mathrm{H}$ & Baltutan & $\begin{array}{l}\text { Davis (1965-1988), } \\
\text { Doğan et al. }(2004)\end{array}$ & $\begin{array}{l}\text { ZI } 1076 \\
\text { ZI } 1173 \\
\text { ZI1166 }\end{array}$ \\
\hline 139 & Lamiaceae & Marrubium vulgare $\mathrm{L}$. & $\mathrm{H}$ & Karaderme & $\begin{array}{l}\text { Davis (1965-1988), } \\
\text { Polunin (1997), } \\
\text { Doğan et al. (2004) }\end{array}$ & ZI 1116 \\
\hline 140 & Lamiaceae & Moluccella laevis $\mathrm{L}$. & $\mathrm{H}$ & Çanakçiçeği & - & ZI 1222 \\
\hline 141 & Lamiaceae & Phlomis bruguieri Desf. & $\mathrm{H}$ & Kabaçalba & $\begin{array}{l}\text { Aydoğdu \& Akan } \\
(2005)\end{array}$ & ZI 1124 \\
\hline
\end{tabular}


Table 1 contd.

\begin{tabular}{|c|c|c|c|c|c|c|}
\hline $\begin{array}{l}\text { Sl. } \\
\text { no. }\end{array}$ & Family & Botanical name & Habit & Vernacular name & Literature cited & $\begin{array}{l}\text { Voucher } \\
\text { no. }\end{array}$ \\
\hline 142 & Lamiaceae & Phlomis kurdica Rech.f. & $\mathrm{H}$ & Gubel & $\begin{array}{l}\text { Ertekin (2002), } \\
\text { Aydoğdu \& Akan } \\
\text { (2005) }\end{array}$ & ZI 1129 \\
\hline 143 & Lamiaceae & $\begin{array}{l}\text { Phlomis pungens Willd. var. } \\
\text { pungens }\end{array}$ & $\mathrm{H}$ & Silvanok & Davis (1965-1988) & ZI 1141 \\
\hline 144 & Lamiaceae & *Rosmarinus officinalis L. & $\mathrm{S}$ & Biberiye & Davis (1965-1988) & ZI 1078 \\
\hline 145 & Lamiaceae & Salvia multicaulis Vahl & $\mathrm{H}$ & Kürtreyhanı & - & ZI 1087 \\
\hline 146 & Lamiaceae & Salvia palaestina Benth. & $\mathrm{H}$ & Sürmelişalba & - & ZI 1134 \\
\hline 147 & Lamiaceae & Salvia syriaca $\mathrm{L}$. & $\mathrm{H}$ & Çevrikotu & - & ZI 1184 \\
\hline 148 & Lamiaceae & Salvia virgata Jacq. & $\mathrm{H}$ & Fatmanaotu & $\begin{array}{l}\text { Davis (1965-1988) } \\
\text { Doğan et al. }(2004)\end{array}$ & ZI 1088 \\
\hline 149 & Lamiaceae & Teucrium polium L. subsp. polium & $\mathrm{H}$ & Acıyavşan & Babacan et al. (2017) & ZI 1137 \\
\hline 150 & Lamiaceae & Ziziphora capitata $\mathrm{L}$. & $\mathrm{H}$ & Anuk & Babacan et al. (2017) & ZI 1013 \\
\hline 151 & Lamiaceae & Ziziphora tenuior $\mathrm{L}$. & $\mathrm{H}$ & Fareotu & - & ZI 1162 \\
\hline 152 & Liliaceae & $\begin{array}{l}\text { Gagea reticulata (Pall.) Schult. } \\
\text { \&Schult.F. }\end{array}$ & $\mathrm{H}$ & Ağyıldızı & Davis (1965-1988) & ZI 1242 \\
\hline 153 & Linaceae & $\begin{array}{l}\text { Linum mисronatum Bertol. subsp. } \\
\text { mucronatum }\end{array}$ & $\mathrm{H}$ & Sarıketen & $\begin{array}{l}\text { Tugay \& Öztürk } \\
(2003)\end{array}$ & $\begin{array}{l}\text { ZI } 1063 \\
\text { ZI } 1229\end{array}$ \\
\hline 154 & Malvaceae & Alcea acaulis (Cav.) Alef. & $\mathrm{H}$ & Hiro & - & ZI 1156 \\
\hline 155 & Malvaceae & Alcea digitata (Boiss.) Alef & $\mathrm{H}$ & Boyluhatmi & Balos \& Akan (2008) & ZI 1126 \\
\hline 156 & Malvaceae & $\begin{array}{l}\text { Alcea hohenackeri (Boiss. \&Huet) } \\
\text { Boiss. }\end{array}$ & $\mathrm{H}$ & Hevur & - & ZI 1098 \\
\hline 157 & Malvaceae & $\begin{array}{l}\text { Alcea striata (DC.) Alef. subsp. } \\
\text { striata }\end{array}$ & $\mathrm{H}$ & Yivlihatmi & Ertekin (2002) & $\begin{array}{l}\text { ZI } 1151 \\
\text { ZI } 1220 \\
\text { ZI } 1183\end{array}$ \\
\hline 158 & Malvaceae & Malva neglecta Wallr. & $\mathrm{H}$ & Çobançöreği & $\begin{array}{l}\text { Davis (1965-1988), } \\
\text { Ertekin (2002), Balos } \\
\text { \& Akan (2008) }\end{array}$ & ZI 1181 \\
\hline 159 & Moraceae & *Ficus carica L. subsp. carica & $\mathrm{T}$ & İncir & $\begin{array}{l}\text { Aydoğdu \& Akan } \\
\text { (2005), Babacan } \\
\text { et al. (2017) }\end{array}$ & ZI 1211 \\
\hline 160 & Moraceae & *Morus alba $\mathrm{L}$. & $\mathrm{T}$ & Dut & - & ZI 1147 \\
\hline 161 & Moraceae & *Morus nigra L. & $\mathrm{T}$ & Karadut & - & ZI 1213 \\
\hline 162 & Oleaceae & *Olea europaea $\mathrm{L}$. & $\mathrm{T}$ & Zeytin & Doğan et al. (2004) & ZI 1238 \\
\hline 163 & Orabanchaceae & Orobanche egyptiaca Pers. & $\mathrm{H}$ & Dinlendiren & - & $\begin{array}{l}\text { ZI } 1037 \\
\text { ZI } 1069\end{array}$ \\
\hline 164 & Orabanchaceae & $\begin{array}{l}\text { Parentucellia latifolia (L.)Caruel } \\
\text { subsp. flaviflora (Boiss.) Hand.- } \\
\text { Mazz }\end{array}$ & $\mathrm{H}$ & Sarıüçdilotu & - & ZI 1042 \\
\hline 165 & Papaveraceae & Fumaria parviflora Lam. & $\mathrm{H}$ & Tarlaşahteresi & - & ZI 1067 \\
\hline 166 & Papaveraceae & $\begin{array}{l}\text { Glaucium grandiflorum Boiss. \& } \\
\text { A. Huet }\end{array}$ & $\mathrm{H}$ & Develalesi & Babacan et al. (2017) & ZI 1119 \\
\hline 167 & Papaveraceae & $\begin{array}{l}\text { Roemeria hybrida (L.) DC. subsp. } \\
\text { hybrida }\end{array}$ & $\mathrm{H}$ & Pitpitotu & - & $\begin{array}{l}\text { ZI } 1029 \\
\text { ZI } 1168\end{array}$ \\
\hline 168 & Papaveraceae & $\begin{array}{l}\text { Papaver clavatum Boiss. et } \\
\text { Hausskn. ex Boiss. }\end{array}$ & $\mathrm{H}$ & Şıkş1k1 & $\begin{array}{l}\text { Davis (1965-1988), } \\
\text { Balos \& Akan (2008) }\end{array}$ & $\begin{array}{l}\text { ZI } 1047 \\
\text { ZI } 1189\end{array}$ \\
\hline
\end{tabular}


Table 1 contd.

\begin{tabular}{|c|c|c|c|c|c|c|}
\hline $\begin{array}{l}\text { Sl. } \\
\text { no. }\end{array}$ & Family & Botanical name & Habit & Vernacular name & Literature cited & $\begin{array}{l}\text { Voucher } \\
\text { no. }\end{array}$ \\
\hline 169 & Pinaceae & $\begin{array}{l}\text { *Pinus nigra J.F. Arnoldsubsp. } \\
\text { pallasiana (Lamb.) Holmboe var. } \\
\text { pallasiana f. pallasiana }\end{array}$ & $\mathrm{T}$ & Karaçam & - & ZI 1194 \\
\hline 170 & Plantaginaceae & Plantago lanceolata $\mathrm{L}$ & $\mathrm{H}$ & Damarlica & $\begin{array}{l}\text { Frenkel (1977), } \\
\text { Davis (1965-1988), } \\
\text { Ertekin (2002), } \\
\text { Doğan } \text { et al. } \text { (2004), } \\
\text { Babacan } \text { et al. (2017) }\end{array}$ & ZI 1191 \\
\hline 171 & Plantaginaceae & Veronica persica Poir. & $\mathrm{H}$ & Circamık & Davis (1965-1988) & ZI 1161 \\
\hline 172 & Poaceae & Aegilops triuncialis $\mathrm{L}$. & $\mathrm{H}$ & Üçkılçık & Doğan et al. (2004) & ZI 1091 \\
\hline 173 & Poaceae & Alopecurus arundinaceus Poir. & $\mathrm{H}$ & Kamıştilkikuyruğu & Davis (1965-1988) & ZI 1237 \\
\hline 174 & Poaceae & Arundo donax $\mathrm{L}$ & S & Karg1 & Doğan et al. (2004) & ZI 1214 \\
\hline 175 & Poaceae & $\begin{array}{l}\text { Avena barbata Pottex Link subsp. } \\
\text { barbata }\end{array}$ & $\mathrm{H}$ & Narinyulaf & $\begin{array}{l}\text { Frenkel (1977), } \\
\text { Poldini (1992), } \\
\text { Doğan et al. (2004) }\end{array}$ & $\begin{array}{l}\text { ZI } 1034 \\
\text { ZI } 1206\end{array}$ \\
\hline 176 & Poaceae & Echinaria capitata (L.) Desf. & $\mathrm{H}$ & Dikenbaşotu & - & ZI 1090 \\
\hline 177 & Poaceae & $\begin{array}{l}\text { Hordeum murinum L. subsp. } \\
\text { glaucum (Steud.) Tzvelev }\end{array}$ & $\mathrm{H}$ & Duvararpası & $\begin{array}{l}\text { Davis (1965-1988), } \\
\text { Polunin (1997), } \\
\text { Doğan et al. (2004) }\end{array}$ & ZI 1197 \\
\hline 178 & Poaceae & Pennisetum orientale Rich. & $\mathrm{H}$ & Fiskiyeotu & - & ZI 1193 \\
\hline 179 & Poaceae & Phalaris paradoxa $\mathrm{L}$. & $\mathrm{H}$ & Topuzlukanyaş & Davis (1965-1988) & ZI 1106 \\
\hline 180 & Poaceae & $\begin{array}{l}\text { Phragmites australis (Cav.) Trin. } \\
\text { ex Steud. }\end{array}$ & $\mathrm{S}$ & Kamış & Doğan et al. (2004) & ZI 1179 \\
\hline 181 & Poaceae & Poa angustifolia $\mathrm{L}$. & $\mathrm{H}$ & Darsalkımotu & - & ZI 1240 \\
\hline 182 & Poaceae & Poa bulbosa $\mathrm{L}$. & $\mathrm{H}$ & Yumrulusalkım & $\begin{array}{l}\text { Frenkel (1977), } \\
\text { Doğan et al. (2004), } \\
\text { Aydoğdu \& Akan } \\
(2005)\end{array}$ & ZI 1021 \\
\hline 183 & Poaceae & $\begin{array}{l}\text { Rostraria berythea (Boiss. } \\
\text { \&Blanche) Holub }\end{array}$ & $\mathrm{H}$ & Maraşgagaotu & Davis (1965-1988) & $\begin{array}{l}\text { ZI } 1053 \\
\text { ZI } 1188\end{array}$ \\
\hline 184 & Poaceae & $\begin{array}{l}\text { Sorghum halepense (L.) Pers.var. } \\
\text { muticum (Hack.) Grossh }\end{array}$ & $\mathrm{H}$ & Ekinsüpürgesi & - & ZI 1131 \\
\hline 185 & Primulaceae & Androsacea maxima $\mathrm{L}$ & $\mathrm{H}$ & Tavukkursağ & - & ZI 1024 \\
\hline 186 & Punicaceae & *Punica granatum $\mathrm{L}$. & $\mathrm{T}$ & Nar & - & ZI 1210 \\
\hline 187 & Resedaceae & Reseda lutea L. var. lutea & $\mathrm{H}$ & Muhabbetçiçeği & $\begin{array}{l}\text { Davis (1965-1988), } \\
\text { Ozturk et al. (1990), } \\
\text { Ozturk \& Ozcelik } \\
\text { (1991), Doğan } \text { et al. } \\
\text { (2004), Babacan et } \\
\text { al. (2017) }\end{array}$ & ZI 1158 \\
\hline 188 & Rosaceae & *Amygdalus communis $\mathrm{L}$. & $\mathrm{T}$ & Badem & $\begin{array}{l}\text { Doğan et al. (2004), } \\
\text { Aydoğdu \& Akan } \\
(2005)\end{array}$ & ZI 1167 \\
\hline 189 & Rosaceae & *Amygdalus orientalis Mill. & $\mathrm{T}$ & Payam & - & ZI 1202 \\
\hline 190 & Rosaceae & *Persica vulgaris Mill. & $\mathrm{T}$ & Şeftali & - & ZI 1055 \\
\hline 191 & Rosaceae & *Pyracantha coccinea M. Roem. & $\mathrm{S}$ & Ateşdikeni & - & ZI 1057 \\
\hline 192 & Rosaceae & Rubus sanctus Schreb. & $\mathrm{S}$ & Böğürtlen & Doğan et al. (2004) & ZI 1120 \\
\hline
\end{tabular}


Table 1 contd.

\begin{tabular}{|c|c|c|c|c|c|c|}
\hline $\begin{array}{l}\text { Sl. } \\
\text { no. }\end{array}$ & Family & Botanical name & Habit & Vernacular name & Literature cited & $\begin{array}{l}\text { Voucher } \\
\text { no. }\end{array}$ \\
\hline 193 & Rosaceae & $\begin{array}{l}\text { Sanguisorba minor L. subsp. } \\
\text { minor }\end{array}$ & $\mathrm{H}$ & Çayırdüğmesi & - & ZI 1014 \\
\hline 194 & Rubiaceae & Callipeltis cucullaris (L.) Stevenv & $\mathrm{H}$ & Nermik & - & ZI 1122 \\
\hline 195 & Rubiaceae & Galium aparine $\mathrm{L}$. & $\mathrm{H}$ & Çobansüzgeci & $\begin{array}{l}\text { Doğan et al, (2004), } \\
\text { Babacan et al. (2017) }\end{array}$ & ZI 1121 \\
\hline 196 & Scrophulariaceae & Scrophularia peyronii Post. & $\mathrm{H}$ & Hilvansıracası & - & ZI 1059 \\
\hline 197 & Scrophulariaceae & Verbascum alepense Benth. & $\mathrm{H}$ & $\begin{array}{l}\text { Halep } \\
\text { slğırkuyruğu }\end{array}$ & Davis (1965-1988) & ZI 1190 \\
\hline 198 & Scrophulariaceae & Verbascum stepporum Hub.-Mor. & $\mathrm{H}$ & $\begin{array}{l}\text { Urfasığır } \\
\text { kuyruğu }\end{array}$ & - & $\begin{array}{l}\text { ZI } 1159 \\
\text { ZI } 1123\end{array}$ \\
\hline 199 & Tamaricaceae & Tamarix parviflora $\mathrm{DC}$. & $S$ & Deli1lgin & - & ZI 1198 \\
\hline 200 & Vitaceae & *Vitis vinifera $\mathrm{L}$. & $\mathrm{Cl}$ & Asma & - & ZI 1209 \\
\hline
\end{tabular}

Note: The numbers after 'ZI' indicate the collection number of voucher specimens of the 1st author. '*' sign = The cultivated plants; $\mathrm{H}=\mathrm{Herb}, \mathrm{Cl}=$ Climber, $\mathrm{S}=$ Shrub, $\mathrm{T}=$ Tree.
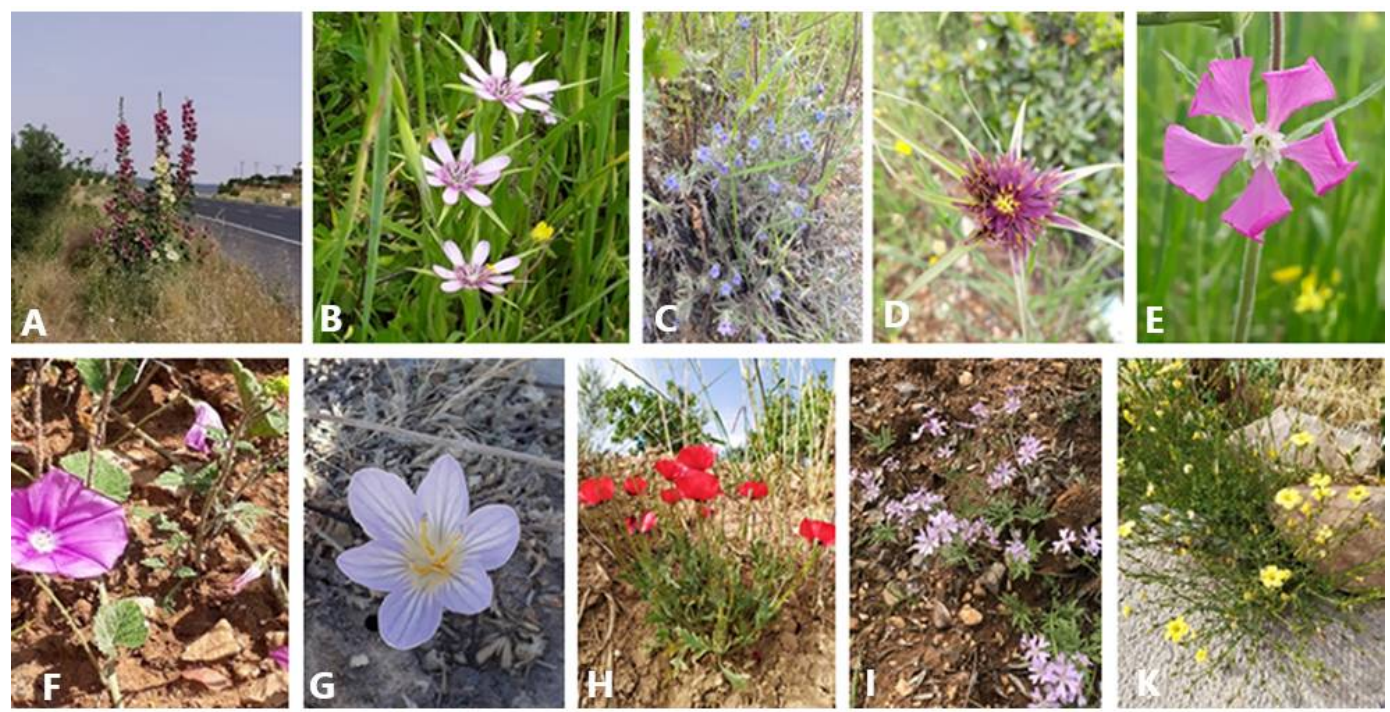

Fig. 2. Some of the common species recorded from the study area; A) Alcea striata subsp. striata, B) Geropogon hybridus, C) Alkanna strigosa, D) Tragopogon porifolius, E) Silene coniflora, F) Convolvulus galaticus, G) Crocus cancellatus subsp. damascenus, H) Papaver clavatum, I) Granium tuberosum, K) Linum mucronatum.

Five geophytes, viz. Poa bulbosa, Crocus cancellatus subsp. damascenus, Iris persica, Ixiolirion tataricum and Biarum carduchorum belonging to different families were determined from the study area. Orobanche aegyptiaca, which belongs to the family Orabanchaceae, has been collected as a parasite species. In the study area, nine endemic taxa have been identified (Table 2), based on which the endemism rate in the study area has been estimated as $4.5 \%$. Since the endemic taxa are generally collected from roadside ruderal areas, they are inevitable under the 
threat in the near future. The life forms of plant species collected from the study area according to Raunkiaer (1934) are presented in Fig. 3. Since the terophytes are very common in arid and semiarid climates, it ranks first in the study area with a rate of $50 \%$. Comparison with other floristic researches in the close vicinity of our study area is given in Table 3.

Table 2. Endemic taxa of the study area and their estimated threatened categories (Ekim, 2000).

\begin{tabular}{llll}
\hline Family name & Endemic plant name & Turkish name & Threat categories \\
\hline Asteraceae & Anthemis pungens Yavin & Yavin & NT \\
Asteraceae & Geropogon hybridus (L.) Sch.Bip. & Melezyemlik & LC \\
Asteraceae & Gundelia armata (Freyn \& Sint.) Firat & Haskenger & EN \\
Caryophyllaceae & Arenaria sabulinea Griseb. ex Fenzl & Firatkumotu & LC \\
Convolvulaceae & Convolvulus galaticus Rost. ex Choisy & Bozsarmaşı̆̆ & LC \\
Fabaceae & Astragalus scabrifolius Boiss. & Gövdesizgeven & CR \\
Fabaceae & Trigonella kotschyi Fenzl & Akboyotu & LC \\
Papaveraceae & Papaver clavatum Boiss. & Şışıkı & LC \\
Scrophulariaceae & Verbascum stepporum Hub.-Mor. & Urfası̆ı̆ırkuyruğu & EN \\
\hline
\end{tabular}

EN: Endangered, NT: Near Threatened, LC: Least Concern, CR:Critically endangered.

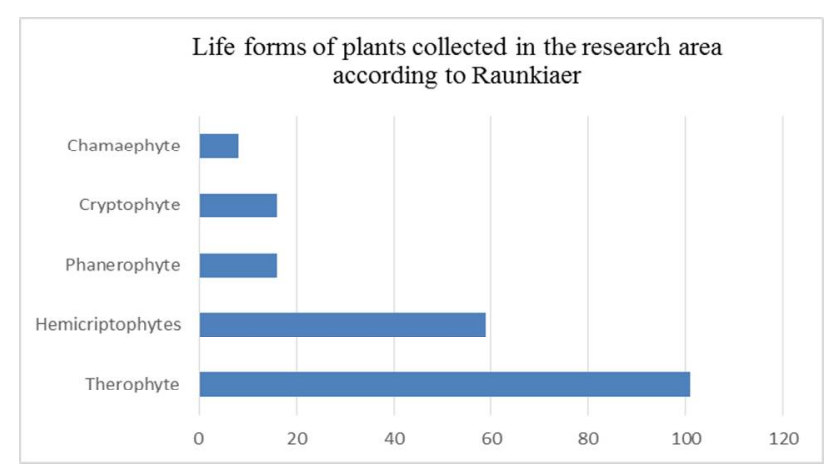

Fig. 3. Raunkiaer (1934)'s life forms spectrum of plant species collected from the study area.

Table 3. Comparison of taxonomic enumeration and endimism rate recorded by this study with those of few previous studies.

\begin{tabular}{lccccc}
\hline & $\begin{array}{c}\text { Our study } \\
(2020)\end{array}$ & $\begin{array}{c}\text { (Aydoğdu and } \\
\text { Akan, 2005) }\end{array}$ & $\begin{array}{c}\text { (Akan and } \\
\text { Ayaz, 2016) }\end{array}$ & $\begin{array}{c}\text { (Akan } \text { et., 2005) } \\
\text { (Atamov et al., }\end{array}$ & 2009) \\
\hline Family no & 41 & 39 & 50 & 47 & 32 \\
Taxa no & 200 & 238 & 226 & 262 & 192 \\
Endemic & 9 & 13 & 6 & 10 & 2 \\
Endemism rate $(\%)$ & 4,5 & $\% 5,4$ & $\% 2,6$ & $\% 3,8$ & $\% 1,04$ \\
\hline
\end{tabular}


The plant species of the study area belonging to different floristic region has been determined. In the study area, the Irano-Turanian elements representing 38\% of the flora are in first place, which is followed by Mediterranean elements with $15 \%$ and Euro-Siberian elements with $1 \%$. The perecent of multi-region or unknown elements is $46 \%$. In the study area, the Iran-Turan region elements are dominant due to the fact that this area is in arid and semi-arid climates. The high number of multi-region and unknown elenments results from the widespread cosmopolitan species and topographic diversity.

According to Flora of Turkey (Davis, 1965-1985), 672 plant taxa occur in Şanliurfa province and only three of them, viz. Nigella orientalis, Xanthium strumarium subsp. strumarium and Anthemis altissima (accepted name is Cota altissima) are are ruderal. During this study, Nigella orientalis was not found but other two taxa were collected.

As shown in Table 3, the taxonomic enumeration of plant taxa recorded by this study is close to that reported by Akan and Ayaz (2016) and Atamov et al. (2009), but lower than that by Aydoğdu and Akan (2005) and Akan et al. (2005). The endemism rate recorded in the study area is higher than that reported by Akan and Ayaz (2016), Akan et al. (2005) and Atamov et al. (2009) (Table 3) and lower than that of Aydoğdu and Akan (2005). In respect to few previous studies (Aydoğdu and Akan, 2005; Akan et al., 2005), a relatively lower number of taxa is found in the study area because this study mostly covers the roadside ruderal plants. The reason for the high rate of endemism in Kalecik mountain (Aydoğdu and Akan, 2005) are its larger natural areas, higher elevation variation and diverse habitats. The density of factories, oil stations, quarries and vineyard houses established along the Şanlıurfa-Bozova road have affected the natural vegetation. Due to the formation of more cultivated areas, uncontrolled factory establishment and construction of vineyard houses etc., degradation of the natural habitats in the region is continuing that should be minimized for the conservation of the plant resources of the study area.

\section{Acknowledgements}

The authors are thankful to Maruf Balos, Mustafa Keskin and Cahit Çeçen for identification of some plant species, to Dr. Erdal Erbil for some technical helpness and to Mahmut İzgördü for field assistants for his service during field surveys.

\section{References}

Abak, F. and Akan, H. 2014. The flora of Asteraceae family in Şanlıurfa/Turkey. Biological Diversity and Conservation 7(1): 68-78.

Acar, C. 2001. Trabzon Yöresi değirmendere ve solaklı havzaları yol şevlerinde yetişen yer örtücü bitkiler. Kafkas Üniversitesi Artvin Orman Fakültesi Dergisi 1: 43-53.

Adıgüzel, N. and Aytaç, Z. 2001. Flora of Ceylanpınar state farm (Şanlıurfa-Turkey). Flora Mediterranea 11: 333-361.

Akan, H. and Ayaz, H. 2016. Gölpınar (Şanlıurfa-Türkiye) mesire yeri florası ve etrafındak iköylerin etnobotanik özellikleri. Bağbahçe Bilim Dergisi 2(3): 19-56.

Akan, H. and Balos, M.M. 2008. Check-list of the genus Biarum Schott in the Flora of Turkey, with a newrecord for Turkey: Biarum syriacum (Spreng.) H. Riedl. Turkish Journal of Botany 32(4): 305-310.

Akan, H., Aytaç, Z. and Ekici, M. 2009. Türkiye'nin Yabani Çemenleri. Ece matbaası, Ankara.

Akan, H., Eker, İ. and Aslan, M. 2004. Kapari (Keber) bitkisinin GAP bölgesindeki ihracat1 ve son populasyon durumu. Ot sistematik Botanik Dergisi 11(1): 105-118.

Akan, H., Kaya, Ö.F., Eker, İ., and Cevheri, C. 2005. The Flora of Kaşmer Dağı (Şanlıurfa), Turkish J. Bot. 29(4): 291-310. 
Altay, V. and Karahan, F. 2017. Ruderal vejetasyon üzerine bir ön çalışma: Antakya (Hatay) örneği. Kilis 7 Araslık Üniversitesi Fen ve Mühendislik Dergisi 1(2): 68-77.

Aslan, M. and Akan, H. 2019. A study of natural woody plants of forest in Şanlıurfa determination of detection and scape values of parks and garden plants. Biological Diversity and Conservation, 12(1): $50-65$

Aslan, M. and Atamov, V. 2006. Flora and vegetation of stonywalls in south-east Turkey (Sanliurfa). Asian Journal of Plant Sciences 5(1): 153-162.

Atamov, V., Aslan, M. and Ayalp, G. 2007a. Flora of Mezra city (Birecik, Sanliurfa- Turkey). Asian Journal of Plant Sciences 6: 225-238.

Atamov, V., Aslan, M. and Aydin, N. 2009. Direkli Tepeleri (Şanlıurfa) Florası. Ot sistematik Botanik Dergisi 6: 97-114.

Atamov, V., Aslan, M., Cevheri, C. and Cetin, E. 2007b. Contribution to the flora of Fatik mountain (Sanliurfa-Turkey). Asian Journal of Plant Sciences 6(1): 1-11.

Aydoğdu, M. and Akan, H. 2005. The flora of Kalecik Mountain (Şanlıurfa), Turkish Journal of Botany 29: $155-174$.

Babacan, E.Y., Vitek, E. and Çakılcıoğlu, U. 2017. Contributions to the flora of Tunceli (Turkey). International Journal of Nature and Life Sciences 1(2): 39-66.

Balos, M.M., and Akan, H. 2008. Flora of the Region between Zeytinbahçe and Akarçay (Birecik, Şanliurfa, Turkey). Turkish Journal of Botany, 32(3): 201-226.

Bozok, F. and Aksoy, A. 2013. Hodul dağı (Nevşehir-Kayseri) ve çevresinin florası. Erciyes Üniversitesi Fen Bilimleri Enstitüsü Fen Bilimleri Dergisi 29(1):10-28.

Cevheri, C. 2011. Çaylarbaşı Şanlıurfa'nın çayır vejetasyonu üzerine floristik bir araştırma. Harran Tarım ve G1da Bilimleri Dergisi 15(4): 9-22.

Çırpıcı, A. 1987. Türkiye'nin flora ve vejetasyonu üzerindeki çalışlmalar. Doğa TU Bot Derg 11(2): 217-232.

Davis, P.H. 1965. Introduction. In: Davis, P.H. (Ed). Flora of Turkey and the East Aegean Islands, Vol. 1. 1st Edition. Edinburgh, Edinburgh University Press, U.K., pp. 1-26.

Davis, P.H. 1965-1985. Flora of Turkey and the East Aegean Islands. Vols. 1-9. Edinburgh: Edinburgh University Pres.

Davis, P.H. 1965-1988. Flora of Turkey and the East Aegean Islands. Vols. 1-9 and Supp. Edinburgh Univ. Press, Edinburgh.

Davis, P.H. 1975. Turkey: present state of floristic knowledge. Coll Int C N R S 235: 93-113.

Davis, P.H., Mill, R.R. and Tan, K. (Eds). 1988. Flora of Turkey and the East Aegean Islands (Supplement1). Vol. 10. Edinburgh: Edinburgh University Pres,

Dogan, Y., Baslar, S., Celik, A., Mert, H.H., and Ozturk, M. 2004. A study of the roadside plants of West Anatolia, Turkey. Natura Croatica: Periodicum Musei Historiae Naturalis Croatici 13(1): 63-80.

Doğan, G. 2009. Elazığ Cip Baraj Gölü-Arındık Köyü arası sahanın florası. Fırat Üniversitesi, Fen Bilimleri Enstitüsü, Elazı̆̆g, Türkiye.

Eker, İ., Koyuncu, M., and Akan, H. 2008. The geophytic flora of Şanlıurfa province, Turkey. Turkish Journal of Botany 32(5): 367-380.

Ekici, M. Akan, H. and Aytac, Z. 2015. Taxonomic revision of Astragalus L. section Onobrychoidei DC. (Fabaceae) in Turkey. Turkish Journal of Botany 39(4): 708-745.

Ekim, T. 2000. Türkiye Bitkileri Kırmızı Kitabı. Türkiye Tabiatını Koruma Derneği ve Yüzüncü Yıl Ün., Ankara, 246 pp.

Ertekin, S. 2002. Karacadağ bitki çeşitliliği. Sürdürülebilir kırsal ve kentsel kalkınma derneği, Ankara.

Frenkel, R.E. 1977. Ruderal vegetation along Some California roadsides. University of California Press, Berkeley-Los Angeles.

Güner, A., Aslan, S., Ekim, T., Vural, M., Babaç, M.T. (Ed). 2012. Türkiye Bitkiler Listesi (Damarlı Bitkiler), Nezahat Gökyiğit Botanik Bahçesi ve Flora Araştırmaları Derneği Yayını, İstanbul. 
Güner, A., Özhatay, N., Ekım, T. and Başer, K.H.C. 2000. Flora of Turkey and The East Aegean Islands (Supp. 2). Vol.11. Edinburgh: Edinburgh University Press.

Güzel, A. 2020. Natural features geography of Şanlıurfa province. The Journal of International Social Research 13(71): 195-2015.

Hamel, A. and Dansereau, P. 1949. L'aspect écologiqu du probleme des mauvaises herbes. Bull. Du Serv. De Biogeographie (Univ. Montreal) 5: 1-45.

Kaya, Ö.F., and Ertekin, A.S. 2009. Flora of the protectedarea at the Tektekdag'ları (Sanliurfa-Turkey). Ot Sistematik Botanik Dergisi 16(2): 79-96.

Kerar, B.A. and Akan, H. 2019. Aktepe ve Zeytinoba Köyleri (Hassa/Hatay-Türkiye) Arasında Kalan Bölgenin Florası ve Etnobotaniği Üzerine Bir Araştırma. Bağbahçe Bilim Dergisi 6(3): 76-96.

Korkut, M.M., Akan, H. and Balos, M.M. 2008. Arat Dağı Florası (Birecik/Şanlıurfa, Türkiye). Selçuk Üniversitesi Fen Fak Fen Derg 2(31): 67-86.

Müdürlüğü, H.G. 2020. The map of Şanlıurfa. https://www.harita.gov.tr/anasayfa, (acces date 13.03.2020).

Ozturk, M. and H. Ozcelik, 1991. Useful plants of East Anatolia. SISKAV, Ankara.

Ozturk, M., Secmen, O., Gemici, Y. and Gork, G. 1990: Plants and Landscape (Aegean Region Turkey). Tukelmat A.S., Izmir.

Parmaksiz, A., Atamov, V., and Aslan, M. 2006. The flora of Osmanbey campus of the Harran University. Journal of Biological Sciences 6(5): 793-804.

Poldini, L. 1992. Environmental alteration and allergophytes. Aerobiologia 8: 127-132.

Polunın, O. 1997. Flowers of Europe. Oxford Univ. Press, Oxford.

Pourrezaei, J., Khajeddin, S.J., Karimzadeh, H.R., Vahabi, M.R., Mozaffarian, V. and Esfahani, M.T. 2017. Roadside flora in arid and semi-arid natural areas (Case study: Northern Khorasan province, Iran). Desert 22(2): 229-237.

Raunkiaer, C., 1934. The Life Forms of Plants and Statistical Plant Geography. Clanrendon Press, Oxford, $632 \mathrm{pp}$.

Şafak, A.S. 2016. Eskișehir ili ruderal vejetasyonunun sintaksonomik analizi. Ankara Üniversitesi, Fen Bilimleri, Biyoloji Anabilim Dalı, Doktora Tezi.

Şafak, S.A. 2015. Ruderal Vejetasyon, Ordu Üniversitesi Bilim ve Teknoloji Dergisi. 5(2): 74-82.

The Plant List, 2013. Version 1.1. Published on the Internet; http://www.theplantlist.org/. Accessed on 19 January 2021.

Thiers, B. 2019. Index Herbariorum: a global directory of public herbaria and associated staff. USA: New York Botanical Garden, New York.

Tugay, O. and Öztürk, F. 2003. Doğu ve Güneydoğu Anadolu Florasına Katkılar. Selçuk Ü., Fen Ed. Fak. Dergisi 22: 7-17.

Türkmen, N., Aslan, M. and Düzenli, A. 2005. Floristic characteristics of the Karkamis Dam reservoir area and its surroundings (Gaziantep-Şanlıurfa: Turkey). Biodiversity \& Conservation 14(10): 2291-2297.

Yalçınalp, E. and Meral, A. 2019. Ruderal Plants in Urban and Sub-Urban Walls and Roofs. Ege Üniversitesi Ziraat Fakültesi Dergisi 56(2): 205-212.

Yeşil, Y., Akalin, E., Akpulat, A. and Vural, C. 2018. Fruit morphology of the genus Pimpinella (Apiaceae) in Turkey. Anales del Jardín Botánico de Madrid 75(2): 3.

Yıldırım, H., Balos, M., Altığlu, Y. and Akan, H. 2016. Biarum aleppicum J.Thiébaut (Araceae): Türkiye için yeni bir tür kaydı. Bahçe Bilim Derg. 3(1): 41-46.

(Manuscript received on 18 November, 2020; revised on 14 May, 2021) 